\title{
Statistical Properties of the T-exponential of Isotropically Distributed Random Matrices.
}

\author{
A.S. Il'yn ${ }^{1,2 *}$ V.A. Sirota ${ }^{1}$, K.P. Zybin ${ }^{1,3}$ \\ 1 P.N.Lebedev Physical Institute of RAS, 119991, Leninskij pr.53, Moscow, Russian Federation \\ 2 National Research Nuclear University MEPhI, 115409, Kashirskoe shosse, 31 Moscow, Russian \\ Federation \\ ${ }^{3}$ National Research University Higher School of Economics, 101000, Myasnitskaya 20, Moscow, \\ Russian Federation
}

\begin{abstract}
A functional method for calculating averages of the time-ordered exponential of a continuous isotropic random $N \times N$ matrix process is presented. The process is not assumed to be Gaussian. In particular, the Lyapunov exponents and higher correlation functions of the T-exponent are derived from the statistical properties of the process.

The approach may be of use in a wide range of physical problems. For example, in theory of turbulence the account of non-gaussian statistics is very important since the non-Gaussian behavior is responsible for the time asymmetry of the energy flow.

Keywords: Lyapunov exponents random matrices T-exponential functional integral stochastic equations turbulence
\end{abstract}

PACS: 02.10Yn 02.50Ey 02.50-r 03.65Db 47.27Gs

\section{Introduction}

Sets of linear stochastic differential equations appear in different physical problems related to quantum mechanics and field theory, turbulence, low temperature physics etc. Their

\footnotetext{
*E-mails: asil72@mail.ru, sirota@lpi.ru, zybin@lpi.ru
} 
formal solution is given by the time-ordered exponential, but calculation of the statistical moments is still a challenge.

The long-time evolution of the time-ordered product of arbitrary (not Gaussian) random matrices in the discrete case was investigated in [1, [2], and [3]. The existence of the Lyapunov spectrum was proved, though no recipe to calculate the Lyapunov exponents or other averages was given. A functional integration method to calculate the averages in the case of Gaussian $\delta$-correlated processes was introduced in [4] and [5].

However, in many applications one needs to calculate the T-exponentials of nonGaussian processes. These are, e.g., field theories with interactions. Also, all the processes that produce non-zero correlators of odd orders, or those in which the Lyapunov spectrum is not even, are knowingly non-Gaussian. This is just what occurs in the theory of magnetic dynamo (see [6]) and in the theory of turbulence: the third-order correlator is related to the energy dissipation rate $([7,8])$, and the asymmetry of the Lyapunov spectrum is necessary to provide the time anisotropy of a turbulent flow $([9,[10])$. Besides, the velocity gradient tensor, which is an important object in the problems related to passive scalar ([11, 12]) and in derivation of scaling exponents $([9,13])$, is proved to have non-Gaussian distribution ([14]). In this paper we propose a functional integration method for calculation the averages of continuous products of $N \times N$ random matrices $A(t)$. The probability distribution of the matrices is assumed to be isotropic, Gaussianity is not required. The method simplifies the functional integrals significantly, in the case of $\delta$-correlation it allows to express all the statistical characteristics of the T-exponential in terms of the moments of $A(t)$. The main idea of the approach is to decompose the matrix product into the rotational and deformational components, and then change the variables. Although the change of variables is non-local (relative to $t$ ), it allows to minimize the non-locality and exclude the time ordering from the path integral. One more important point is that we use a cumulant function to analyze the $\delta$-processes. This allows to avoid renormalizations, and to get a simple expressions for all the averages. 


\section{T-exponential of a random process}

Let $A(t), 0 \leq t \leq T$ be a random process taking on a value of $N \times N$ real matrices. Its statistics is defined by the measure

$$
D A P[A] \equiv \prod_{0 \leq t \leq T} \prod_{k, p=1}^{N} d A_{k p} P[A],
$$

where $P[A]$ is the probability density functional.

Consider the random matrices $Q(t)$ that satisfy the equation

$$
\partial_{t} Q=Q A, Q(0)=\hat{1}
$$

The formal solution to the equation can be written in terms of the anti-chronological exponential ([15]):

$$
Q(t)=\stackrel{+}{T} \exp \left(\int_{0}^{t} A(\tau) d \tau\right)=\sum_{n} \frac{1}{n !} \int_{0}^{t} d \tau_{1} \ldots d \tau_{n} \stackrel{+}{T}\left(A\left(\tau_{1}\right) \ldots A\left(\tau_{n}\right)\right)
$$

where

$$
\stackrel{+}{T}\left(A\left(\tau_{1}\right) \ldots A\left(\tau_{n}\right)\right)=A\left(\tau_{i_{1}}\right) \ldots A\left(\tau_{i_{n}}\right), \quad \tau_{i_{1}} \leq \ldots \leq \tau_{i_{n}}
$$

is the antichronological product operator. The alternative way to describe the solution is the Volterra multiplicative integral ([16]):

$$
Q(t)=\prod_{\tau=0}^{t}(1+A(\tau) d \tau)
$$

Thus, the T-exponent is equivalent to the infinite matrix product.

We are interested in the averages

$$
\langle F[Q]\rangle=\int D A P[A] F[Q],
$$

where $F[Q]$ is some functional. These expressions contain the T-exponent which is not an easy object to deal with. Below we simplify them and reduce, in the case of isotropically distributed processes, to the path integrals of some exponents without any time-ordering.

\section{Change of variables}

We make the Iwasawa decomposition of the matrix $Q$ :

$$
Q=z d R
$$


where $z$ is an upper triangular matrix with diagonal elements equal to $1, d$ is a diagonal matrix, $R$ is an orthogonal matrix: $R_{j i} R_{j k}=\delta_{i k}$.

We recall that $Q$ as well as $z, d, R$ is a function of time. From (1) it follows $A=Q^{-1} \partial_{t} Q$; thus, Eq. (5) produces the decomposition of $A$ :

$$
A=R^{T} X R, \quad X=\rho+\zeta+\theta
$$

where

$$
\begin{gathered}
\rho=d^{-1} \partial_{t} d, \quad \zeta=d^{-1} z^{-1}\left(\partial_{t} z\right) d \\
\theta=\left(\partial_{t} R\right) R^{T}
\end{gathered}
$$

We see that $\rho$ is diagonal, $\zeta$ is an upper triangular matrix with zeroes in the main diagonal, and $\theta$ is antisymmetric:

$$
\rho=\operatorname{diag}\left(\rho_{1}, \ldots, \rho_{N}\right), \quad \theta_{i j}=-\theta_{j i}, \quad \zeta_{i j}=0 \text { if } i \geq j
$$

Now we consider $X(t)$ as independent functional variables; then (66) should be understood as

$$
A=R^{T}[X] X R[X]
$$

Where $R[X]$ is determined by (8) :

$$
R(t)=T \exp \left(\int_{0}^{t} \theta(\tau) d \tau\right)
$$

Note that $R$ depends only on the $\theta$-component of $X$; this will simplify further calculations significantly. This also makes it possible to separate the rotational part of $Q$ from deformation.

The averages (41) can be rewritten as

$$
\langle F[Q]\rangle=\int D X J[X] P\left[R^{T}[X] X R[X]\right] F[Q[X]]
$$

The Jacobian $J[X]$ will be calculated in the next section.

The advantage of this change of variables is in dealing with a simple 'rotational' Texponent (10), instead of the complicated T-exponent (2). As we will see below, this one does also vanish in the isotropic case. 


\section{The Jacobian}

In this section, we calculate the functional Jacobian

$$
J=\operatorname{Det}\left(\frac{\delta A_{i j}(t)}{\delta X_{k p}\left(t^{\prime}\right)}\right)
$$

First, to simplify the notations, we introduce the multiindices (hereafter denoted by Greek letters):

$$
\alpha \equiv(i, j) ; \quad A_{\alpha} \equiv A_{i j}, \quad X_{\beta} \equiv X_{k p}, \quad \alpha, \beta, \ldots=1 . . N^{2}
$$

The transformation (9) can be presented as

$$
A_{\alpha}=\Re_{\alpha \beta}[X] X_{\beta}
$$

where $\Re_{\alpha \beta}[X]$ is the $N^{2} \times N^{2}$ matrix that satisfies the relation:

$$
\Re_{(i j),(k p)}=R_{k i} R_{p j}
$$

(This corresponds to multiplying $X$ by $\Re$ from right and by $R^{T}$ from left). The functional derivative of (12) is equal to

$$
\frac{\delta A_{\alpha}(t)}{\delta X_{\beta}\left(t^{\prime}\right)}=\Re_{\alpha \beta}(t) \delta\left(t-t^{\prime}\right)+\frac{\delta \Re_{\alpha \gamma}(t)}{\delta X_{\beta}\left(t^{\prime}\right)} X_{\gamma}(t)
$$

Now, as it follows from (13) and (10), $\Re(t)$ is determined completely by the function $\theta(\tau)$ at $\tau \leq t$. Thus, $\Re_{\alpha \beta}(t)$ does not depend on $X_{\gamma}\left(t^{\prime}\right)$ if $t<t^{\prime}$.

According to (14), the Jacobian is a block-triangular matrix:

$$
\left(\frac{\delta A_{\alpha}(t)}{\delta X_{\beta}\left(t^{\prime}\right)}\right)=0, \quad t<t^{\prime}
$$

Consequently, its determinant is a continuous product of the determinants of the diagonal $\left(t=t^{\prime}\right) N^{2} \times N^{2}$ blocks:

$$
J \equiv \operatorname{Det}\left(\frac{\delta A_{\alpha}(t)}{\delta X_{\beta}\left(t^{\prime}\right)}\right)=\prod_{0 \leq t \leq T} \tilde{J}(t), \quad \tilde{J}(t)=\operatorname{det}\left(\frac{\delta A_{\alpha}(t)}{\delta X_{\beta}(t)}\right)
$$

Now, let us calculate $\tilde{J}(t)$. From (14) it follows

$$
\tilde{J}(t)=\operatorname{det}\left(\Re_{\alpha \beta}(t) \delta(0)+\frac{\delta \Re_{\alpha \gamma}(t)}{\delta X_{\beta}(t)} X_{\gamma}(t)\right)
$$


Note that $\Re_{\alpha \beta}$ is an orthogonal matrix, since

$$
\Re_{\mu \alpha} \Re_{\mu \beta} \equiv \Re_{(i j),(k p)} \Re_{(i j),(m n)}=R_{k i} R_{p j} R_{m i} R_{n j}=\delta_{k m} \delta_{p n}=\delta_{(k p),(m n)}=\delta_{\alpha \beta}
$$

So, $\operatorname{det} \Re=1$, and one can multiply the bracketed expression by $\Re^{T}$. Then, assuming $\delta(0)=(d t)^{-1}$ and omitting the insufficient normalization multipliers, we obtain

$$
\tilde{J}(t)=\operatorname{det}\left(\delta_{\alpha \beta}+\Re_{\mu \alpha} \frac{\delta \Re_{\mu \gamma}}{\delta X_{\beta}} X_{\gamma} d t\right)=\exp \left(\operatorname{tr}\left(\Re_{\mu \alpha} \frac{\delta \Re_{\mu \gamma}}{\delta X_{\beta}} X_{\gamma}\right) d t\right)
$$

From (15) it follows

$$
J=\exp \left(\int_{0}^{T} G d t\right), \quad G=\Re_{\mu \alpha} \frac{\delta \Re_{\mu \gamma}}{\delta X_{\alpha}} X_{\gamma}
$$

To calculate $G$, we return from multiindices to usual matrix notations:

$$
G=R_{k i} R_{p j} \frac{\delta\left(R_{n i} R_{m j}\right)}{\delta X_{k p}} X_{n m}
$$

Now we need to calculate the variation derivative $\frac{\delta R_{n m}(t)}{\delta X_{k p}(t)}$ at coinciding time. The $R(t)$ dependence of $X(t)$ is determined by (16), (10). Thus, of all the $N^{2}$ components of the matrix $X, R$ depends on $N(N-1) / 2$ independent components of $\theta_{i j}$ only.

Since $X_{i j}$ coincides with $\theta_{i j}$ as $i>j$, we get

$$
\frac{\delta \theta_{i j}(t)}{\delta X_{k p}(t)}=\left\{\begin{array}{lll}
\delta_{i k} \delta_{j p} & \text { if } & k>p \\
0 & \text { if } & k \leq p
\end{array}\right.
$$

Hence, $\frac{\delta R_{i j}(t)}{\delta X_{k p}(t)}=0$ if $k \leq p$.

For the rest, we use the Volterra presentation (3) of the T-exponent (10):

$$
\begin{aligned}
\left.\frac{\delta R_{i j}(t)}{\delta \theta_{k p}(t)}\right|_{k>p}= & \frac{\delta}{\delta \theta_{k p}(t)}\left(\prod_{\tau=t}^{0}(1+\theta(\tau) d \tau)\right)_{i j}= \\
& \int_{0}^{t}\left(\prod_{\tau=t}^{\tau^{\prime}}(1+\theta(\tau) d \tau)\right)_{i m} \frac{\delta \theta_{m n}\left(\tau^{\prime}\right)}{\delta \theta_{k p}(t)} d \tau^{\prime}\left(\prod_{\tau=\tau^{\prime}}^{0}(1+\theta(\tau) d \tau)\right)_{n j}
\end{aligned}
$$

Further,

$$
\frac{\delta \theta_{m n}\left(\tau^{\prime}\right)}{\delta \theta_{k p}(t)}=\delta\left(\tau^{\prime}-t\right)\left(\delta_{m k} \delta_{n p}-\delta_{m p} \delta_{n k}\right),
$$

and we make use of the time-ordering and note that the derivative can only make a non-zero contribution when acting on the first $\left(\tau^{\prime}=t\right)$ multiplier. Then the rest of the multipliers 
make $R$ again, and $\int_{0}^{t} \delta\left(t-\tau^{\prime}\right) d \tau^{\prime}=\frac{1}{2}$ because $t$ is the boundary of the integral. (Effectively, only one half of the delta-function is integrated.) The integral becomes more compact:

$$
\left.\frac{\delta R_{i j}(t)}{\delta \theta_{k p}(t)}\right|_{k>p}=\frac{1}{2}\left(\delta_{i k} \delta_{m p}-\delta_{i p} \delta_{m k}\right)\left(\prod_{\tau=t}^{0}(1+\theta(\tau) d \tau)\right)_{m j}=\frac{1}{2}\left(\delta_{i k} \delta_{m p}-\delta_{i p} \delta_{m k}\right) R_{m j}
$$

Thus,

$$
\frac{\delta R_{i j}(t)}{\delta X_{k p}(t)}= \begin{cases}\frac{1}{2}\left(\delta_{i k} \delta_{m p}-\delta_{i p} \delta_{m k}\right) R_{m j}, & k>p \\ 0, & k \leq p\end{cases}
$$

As we substitute this into (17) and recall that $R^{T} R=I$, all the matrices $R$ are cancelled, and we get

$$
G=\sum_{k>p} \frac{\left(X_{k k}-X_{p p}\right)}{2}=\operatorname{tr}\left(\eta_{0} X\right)
$$

where

$$
\left(\eta_{0}\right)_{k p}=\frac{2 k-1-N}{2} \delta_{k p}
$$

From (16) we eventually have

$$
J[X]=\exp \left(\int_{0}^{T} \operatorname{tr}\left(\eta_{0} X(t)\right) d t\right)
$$

\section{$5 \quad$ Isotropic processes}

Now we express the probability functional in the form

$$
P[A]=\exp \left(-\int_{0}^{T} L\left(A, \partial_{t} A, \partial_{t}^{2} A, \ldots\right) d t\right)
$$

where $L$ is the Lagrangian of the process.

The change of variables (9) transforms $P[A] D A$ to $P_{X}[X] D X$ where

$$
P_{X}[X]=P\left[R^{T} X R\right] J=\exp \left(-\int_{0}^{T} L_{X} d t+\int_{0}^{T} \operatorname{tr}\left(\eta_{0} X\right) d t\right)
$$

Here

$$
L_{X}=L\left(R^{T} X R, \partial_{t}\left(R^{T} X R\right), \partial_{t}^{2}\left(R^{T} X R\right), \ldots\right)
$$

contains generally not only derivatives but also integrals of $X(t)$, since $R[X]$ contains the T-exponent (10). Hereafter, we restrict our consideration with the isotropic processes:

$$
P\left[O^{T} A O\right]=P[A] \quad \forall O \in S O(N)
$$


(This means that statistical properties of the process would not change under the global rotation of the reference frame.) We will show that for such processes, $L_{X}$ does not contain the time integrals.

The condition (20) implies that the matrix $A$ and its time derivatives can contribute to $L$ only in scalar combinations like

$$
\operatorname{tr}\left(A^{b_{0}} \ldots\left(\partial_{t}^{a_{1}} A\right)^{b_{1}}\left(\partial_{t}^{a_{2}} A^{T}\right)^{b_{2}} \ldots\right)
$$

But from (8) it follows

$$
\partial_{t} R=\theta R, \quad \partial_{t}^{2} R=\left(\partial_{t} \theta+\theta^{2}\right) R, \quad \partial_{t} R^{T}=R^{T} \theta^{T}, \ldots
$$

accordingly,

$$
\partial_{t}\left(R^{T} X R\right)=R^{T}\left(\theta^{T} X+\partial_{t} X+X \theta\right) R, \quad \partial_{t}^{a}\left(R^{T} X R\right)=R^{T}(\ldots) R
$$

Hence, as we substitute $R^{T} X R$ for $A$ in (21), all the $R^{T}$ are multiplied by $R$ and vanish. As a result, $L_{X}$ contains scalar combinations of $X$ and $\theta$ and their derivatives, and does not contain $R$. According to ([6), the matrix $\theta$ is itself a function of $X$, so

$$
L_{X}[X]=L_{X}\left(X, \partial_{t} X, \partial_{t}^{2} X, \ldots\right)
$$

Thus, the new Lagrangian $L_{X}$ is a differential function of the matrix $X$.

\section{Isotropic $\delta$-processes.}

There is an important particular case of absence of correlation between the values of $A$ at different time moments:

$$
P[A]=\exp \left(-\int_{0}^{T} L(A) d t,\right)
$$

the Lagrangian $L$ being a function of $A$ only, not of its derivatives. We also demand that $A$ is isotropic (20). Then $A$ can contribute to $L$ only as a part of invariant combinations:

$$
L(A)=L\left(\operatorname{tr} A, \operatorname{tr} A^{2}, \operatorname{tr} A A^{T}, \operatorname{tr} A^{3} \ldots\right)
$$

The change of variables $A \mapsto X$ results in the substitution $X$ for $A$, since all the $R$ and $R^{T}$ vanish. Thus,

$$
L_{X}[X]=\left.L(A)\right|_{A=X}
$$


and

$$
P_{X}[X]=\exp \left(-\int_{0}^{T} L(X) d t+\int_{0}^{T} \operatorname{tr}\left(\eta_{0} X\right) d t\right)
$$

To get the correlation functions of $X$, one now just has to calculate the functional integral (11):

$$
\left\langle X_{i j}\left(t_{1}\right) \ldots X_{k p}\left(t_{n}\right)\right\rangle=N^{\prime} \int D X X_{i j}\left(t_{1}\right) \ldots X_{k p}\left(t_{n}\right) P_{X}[X]
$$

where

$$
N^{\prime}=\left(\int D X \exp \left(-\int_{0}^{T} L(X) d t+\int_{0}^{T} \operatorname{tr}\left(\eta_{0} X\right) d t\right)\right)^{-1}
$$

Since $X$ is an integration variable, it can be changed to $A$ in the right-hand side of the expression. Note that the integral does not contain T-exponents.

To simplify the calculations, one introduces the characteristic functional of a random process:

$$
Z[\eta(t)]=\left\langle\exp \left(\int_{0}^{T} \operatorname{tr}(\eta(t) A(t)) d t\right)\right\rangle=\int D A P[A] \exp \left(\int_{0}^{T} \operatorname{tr}(\eta A) d t\right)
$$

instead of $P[A]$. The averages can then be expressed as

$$
\left\langle A_{i j}\left(t_{1}\right) \ldots A_{k p}\left(t_{n}\right)\right\rangle=\left.\frac{1}{Z} \frac{\delta}{\delta \eta_{i j}\left(t_{1}\right)} \ldots \frac{\delta}{\delta \eta_{k p}\left(t_{n}\right)} Z\right|_{\eta(t)=0}
$$

To determine the statistics of $Q$ (2), (4), we have to calculate different correlators of $X$. From (24) it then follows that for $\delta$-processes the correlation function of $X$ can also be written in terms of the same $Z[\eta]$ :

$$
\left\langle X_{i j}\left(t_{1}\right) \ldots X_{k p}\left(t_{n}\right)\right\rangle=\left.\frac{1}{Z} \frac{\delta}{\delta \eta_{i j}\left(t_{1}\right)} \ldots \frac{\delta}{\delta \eta_{k p}\left(t_{n}\right)} Z\right|_{\eta(t)=\eta_{0}}
$$

The only difference from (26) is that the expression is calculated at the point $\eta(t)=\eta_{0}$ instead of $\eta(t)=0$.

It is convenient to introduce the generating functional for connected correlation functions defined by

$$
Z[\eta]=e^{W[\eta]}
$$


The normalization requires $W[\eta(t)=0]=0$. Since $Z[\eta]$ is a Fourier transform of $P[A]$, the isotropy (20) of $P[A]$ leads to isotropy of $Z[\eta]$ and $W[\eta]$ :

$$
W\left[O^{T} \eta O\right]=W[\eta] \quad \forall O \in S O(N)
$$

Furthermore, if $P[A]$ is a $\delta$-process $(22), Z$ is also a continuous product of independent multipliers, and

$$
W[\eta(t)]=\int_{0}^{T} w(\eta(t)) d t
$$

The function $w(\eta)$ is called a cumulant function (see, e.g., [17]). Via this function, one can calculate any of the correlators by consecutive differentiation:

$$
\left\langle A_{i j}\left(t_{1}\right) \ldots A_{k p}\left(t_{n}\right)\right\rangle=\left.\frac{\delta}{\delta \eta_{i j}\left(t_{1}\right)} \ldots \frac{\delta}{\delta \eta_{k p}\left(t_{n}\right)} \exp \left(\int_{0}^{T} w(\eta) d t\right)\right|_{\eta(t)=0}
$$

The first term of each correlator,

$$
\left\langle A_{i j}\left(t_{1}\right) \ldots A_{k p}\left(t_{n}\right)\right\rangle_{c}=\frac{\partial^{n} w}{\partial \eta_{i j} \ldots \partial \eta_{k p}}(0) \delta\left(t_{2}-t_{1}\right) \delta\left(t_{3}-t_{1}\right) \ldots \delta\left(t_{n}-t_{1}\right),
$$

is called the connected correlation function and corresponds to the connected diagram (as $t_{1}, \ldots, t_{n}$ are represented by $n$ points, and $\delta$-functions make connections between them). The next terms contain the products of lower-order connected correlation functions (and hence, smaller sets of $\delta$-functions) and correspond to non-connected diagrams.

By analogy to (27), all the correlators of the $X$-variables can easily be obtained from the same expressions as these for $A$-variables by changing the point where the derivatives are taken. For the connected correlation functions we get:

$$
\left\langle X_{i j}\left(t_{1}\right) \ldots X_{k p}\left(t_{n}\right)\right\rangle_{c}=\frac{\partial^{n} w}{\partial \eta_{i j} \ldots \partial \eta_{k p}}\left(\eta_{0}\right) \delta\left(t_{2}-t_{1}\right) \delta\left(t_{3}-t_{1}\right) \ldots \delta\left(t_{n}-t_{1}\right)
$$

\section{The Lyapunov spectrum}

In this section we use the method described above to calculate the averages of the matrix elements $X_{i j}$ in the case of isotropic $\delta$-processes. From (31) it follows

$$
\left\langle X_{s q}\right\rangle=\frac{\partial}{\partial \eta_{s q}} w\left(\eta_{0}\right)
$$


First, we show that the non-diagonal matrix elements are equal to zero. Actually, since $w(\eta)$ is isotropic, it depends on a combination of traces:

$$
w(\eta)=w\left(\operatorname{tr} \eta, \operatorname{tr} \eta^{2}, \operatorname{tr} \eta \eta^{T}, \operatorname{tr} \eta^{3} \ldots\right)
$$

The derivative of each trace is

$$
\frac{\partial}{\partial \eta_{s q}} \operatorname{tr}\left(\eta \ldots \eta^{T} \ldots\right)=\sum \delta_{i s} \delta_{j q}\left(\eta \ldots \eta^{T} \ldots\right)_{j i}
$$

Since the derivative is taken at $\eta_{k p}=\left(\eta_{0}\right)_{k p} \sim \delta_{k p}$ (see (18)), we get $\delta_{i s} \delta_{j q} \delta_{i j}$ in each term, and non-diagonal elements vanish:

$$
\left\langle X_{s q}\right\rangle=\frac{\partial}{\partial \eta_{s q}} w\left(\eta_{0}\right)=0, \quad s \neq q
$$

The averages of the diagonal components,

$$
\left.\lambda_{s}=\left\langle X_{s s}\right\rangle=\left\langle\rho_{s}\right\rangle \quad \text { (no summation }\right)
$$

are called the Lyapunov exponents. The set of $\lambda_{s}$ is an important statistical characteristic of a process. It is used in many physical applications (e.g., to describe the separation of trajectories in a turbulent flow).

Before we proceed to these calculations, we make one useful deduction:

For the values $\lambda_{s}$, as well as other diagonal averages, the function $w(\eta)$ in (31), (32) can be replaced by the diagonal cumulant function $w_{d}$ where all the non-zero elements are set equal to zeros:

$$
w_{d}\left(\eta_{11}, \ldots, \eta_{N N}\right)=\left.w(\eta)\right|_{\eta_{k p}=0, k \neq p}
$$

This is caused by the diagonality of $\eta_{0}$ so in (24) one can make an integration over the non-diagonal elements to get the 'diagonal' probability function

$$
P_{d}\left[A_{11}, \ldots, A_{N N}\right]=\int \prod_{k \neq p} D A_{k p} \exp \left(-\int_{0}^{T} L(A) d t\right)
$$

The cumulant function (35) corresponds to this PDF.

\subsection{Gaussian process}

Let the probability distribution of the process $A(t)$ be Gaussian:

$$
P[A]=\exp \left(-\frac{1}{4} \int_{0}^{T} A_{i j} D_{i j k p}^{-1} A_{k p} d t\right),
$$


where $D_{i j k p}$ is determined by the pair correlation function,

$$
2 D_{i j k p} \delta\left(t-t^{\prime}\right)=\left\langle A_{i j}(t) A_{k p}\left(t^{\prime}\right)\right\rangle
$$

Isotropy requires

$$
D_{i j k p}=a \delta_{i j} \delta_{k p}+b \delta_{i k} \delta_{j p}+c \delta_{i p} \delta_{j k}
$$

Here $a, b, c$ are constants (they must satisfy the condition $P[A]>0$ for any $A$ ). The cumulant function corresponding to (36) is

$$
w(\eta)=\eta_{i j} D_{i j k p} \eta_{k p}=a(t r \eta)^{2}+b \operatorname{tr} \eta \eta^{T}+c t r \eta^{2}
$$

From (32) we then get

$$
\lambda_{s}^{G}=D(2 s-1-N), \quad D=b+c
$$

We note that the Gaussian spectrum is antisymmetric relative to the change $s \rightarrow N-s+1$; in particular,

$$
\sum_{s} \lambda_{s}^{G}=0
$$

In many applications one needs the additional restriction $\operatorname{tr} A=0$. From $\left\langle(\operatorname{tr} A)^{2}\right\rangle=0$ it then follows 1

$$
N a+b+c=0
$$

Since $a$ does not contribute to $\lambda_{s}$, this condition does not affect the spectrum (38).

To describe the statistics of $X_{s s}=\rho_{s}$ more accurately, one can also calculate its meansquare deviation from the average: denote

$$
\xi_{s}=\rho_{s}-\lambda_{s}
$$

then

$$
\left\langle\xi_{s}\left(t_{1}\right) \xi_{q}\left(t_{2}\right)\right\rangle=\left\langle\rho_{s}\left(t_{1}\right) \rho_{q}\left(t_{2}\right)\right\rangle_{c}=\left\langle\rho_{s}\left(t_{1}\right) \rho_{q}\left(t_{2}\right)\right\rangle-\lambda_{s} \lambda_{q}
$$

According to (31),

$$
\left\langle\xi_{s}\left(t_{1}\right) \xi_{q}\left(t_{2}\right)\right\rangle=D_{s q} \delta\left(t_{1}-t_{2}\right), \quad D_{s q}=\frac{\partial}{\partial \eta_{s s}} \frac{\partial}{\partial \eta_{q q}} w\left(\eta_{0}\right)
$$

\footnotetext{
${ }^{1}$ The matrix $D_{i j k p}^{-1}$ then becomes singular, which formally corresponds to appearance of the multiplier $\prod_{t} \delta(\operatorname{tr} A(t))$.
} 
Simplifying the calculation by using only diagonal components of $w(\eta)$ in accordance with (35), for the Gaussian function (37) we have

$$
D_{s q}^{G}=2\left(D \delta_{s q}+a\right)
$$

Unlike the Lyapunov exponents, the dispersion of $\rho_{s}$ depends on the distribution of $\operatorname{tr} A$. In the case of traceless Gaussian matrices we get the well-known relation derived by [12]:

$$
\left\langle\xi_{s}^{G}\left(t_{1}\right) \xi_{q}^{G}\left(t_{2}\right)\right\rangle=2 D\left(\delta_{s q}-\frac{1}{N}\right) \delta\left(t_{1}-t_{2}\right)
$$

\subsection{Non-Gaussian process}

Let now $A(t)$ be a non-Gaussian isotropic $\delta$-process. As we have seen in the beginning of the section, for diagonal components of $\left\langle X_{s q}\right\rangle$ one can use (32) with $w(\eta)$ replaced by its diagonal part (35):

$$
\lambda_{s}=\left.\frac{\partial}{\partial \eta_{s s}} w_{d}\left(\eta_{11} \ldots \eta_{N N}\right)\right|_{\eta=\eta_{0}}
$$

The isotropy condition (33) means that $w_{d}$ must be a function of combinations $\sum_{s}\left(\eta_{s s}\right)^{n}$ with different $n$ :

$$
w_{d}\left(\eta_{11} \ldots \eta_{N N}\right)=f\left(\sum_{s}\left(\eta_{s s}\right), \sum_{s}\left(\eta_{s s}\right)^{2}, \sum_{s}\left(\eta_{s s}\right)^{3}, \ldots\right)
$$

Decomposing $w_{d}$ into symmetric and antisymmetric (relative to $\eta \rightarrow-\eta$ ) parts, we get

$$
w_{d}(\eta)=w_{d}^{+}(\eta)+w_{d}^{-}(\eta), \quad w_{d}^{ \pm}(\eta)=\frac{w_{d}^{+}(\eta) \pm w_{d}^{-}(-\eta)}{2}
$$

One can see from (30) that the symmetric part of $w_{d}$ contributes to the connected even order correlation funcitons of $A$, and $w_{d}^{-}$contributes to those of odd orders. Furthermore, we decompose

$$
\lambda_{s}=\lambda_{s}^{-}+\lambda_{s}^{+}
$$

here $\lambda_{s}^{+}$is produced by the antisymmetric part of $w_{d}$, and vice versa:

$$
\lambda_{s}^{ \pm}=\left.\frac{\partial}{\partial \eta_{s s}} w_{d}^{\mp}\right|_{\eta_{0}}
$$

Then, symmetric properties of $w_{d}$ cause the symmetric properties of $\lambda_{s}$ relative to $\eta_{0} \rightarrow-\eta_{0}$, i.e., $s \rightarrow N+1-s$ :

$$
\lambda_{N+1-s}^{ \pm}= \pm \lambda_{s}^{ \pm}
$$


Hence, the symmetric part $\lambda_{s}^{+}$is determined by the odd-order correlators of $A$, and the asymmetric part $\lambda_{s}^{-}$depends on the even-order correlators 2 This is an important feature of the random processes: e.g., in theory of turbulence it is connected with the asymmetry of a turbulent flow relative to the change of time direction $([9,10])$.

If the matrices $A$ (and hence, $X$ ) are assumed to be traceless, we have

$$
\sum_{s} \lambda_{s}^{+}=\sum_{s} \lambda_{s}=\sum_{s}\left\langle X_{s s}\right\rangle=0
$$

Generally, the Lyapunov spectrum is determined by the choice of $f$ in (42). Here we analyze one particular (important for physical applications) case of traceless $3 \times 3$ matrices $A(t)$ with distribution close to the Gaussian.

For $N=3$ and $\operatorname{tr} A=0$, the Lyapunov spectrum takes the form

$$
\left\{\begin{array}{l}
\lambda_{1}=-\Delta-\frac{\lambda_{2}}{2} \\
\lambda_{2} \\
\lambda_{3}=\Delta-\frac{\lambda_{2}}{2}
\end{array}\right.
$$

According to (43), $\lambda_{2}$ is determined by the odd, and $\Delta$ by the even part of $w_{d}$. If we restrict (42) to the first two terms, the traceless condition

$$
\sum_{s} \frac{\partial}{\partial \eta_{s s}} w_{d}=0
$$

gives

$$
\begin{aligned}
w_{d}(\eta) & =D\left(\sum_{q} \eta_{q q}^{2}-\frac{1}{3}\left(\sum_{q} \eta_{q q}\right)^{2}\right) \\
& +F\left(\left(\sum_{q} \eta_{q q}^{2}\right)\left(\sum_{q} \eta_{q q}\right)-\sum_{q} \eta_{q q}^{3}-\frac{2}{9}\left(\sum_{q} \eta_{q q}\right)^{3}\right)
\end{aligned}
$$

The coefficient $F$ is called the asymmetry coefficient of the process; in accordance with (30), it determines the third-order correlation of $A$, for example

$$
\left\langle A_{11}\left(t_{1}\right) A_{11}\left(t_{2}\right) A_{11}\left(t_{3}\right)\right\rangle=-\frac{4}{3} F \delta\left(t_{1}-t_{2}\right) \delta\left(t_{1}-t_{3}\right)
$$

From (41) it follows

$$
\lambda_{s}=2 D(s-2)+2 F\left(1-\frac{3}{2}(s-2)^{2}\right)
$$

So,

$$
\Delta=2 D \quad \lambda_{2}=2 F
$$

\footnotetext{
${ }^{2}$ Note that in the Gaussian case the Lyapunov spectrum is odd because there are no non-zero odd-order correlators.
} 
For the second-order correlator (39), (401) we obtain

$$
D_{s q}=2 D\left(\delta_{s q}-\frac{1}{3}\right)+2 F(s+q-4)\left(1-\frac{3}{2} \delta_{s q}\right)
$$

The first term in this equation is Gaussian, the second term corresponds to the main nonGaussian contribution.

In non-Gaussian process, there is also the third-order connected correlator:

$$
\left\langle\xi_{s}\left(t_{1}\right) \xi_{q}\left(t_{2}\right) \xi_{p}\left(t_{3}\right)\right\rangle=\left\langle\rho_{s}\left(t_{1}\right) \rho_{q}\left(t_{2}\right) \rho_{p}\left(t_{3}\right)\right\rangle_{c}=F_{s q p} \delta\left(t_{1}-t_{2}\right) \delta\left(t_{1}-t_{3}\right)
$$

where

$$
F_{s q p}=\frac{\partial}{\partial \eta_{s s}} \frac{\partial}{\partial \eta_{q q}} \frac{\partial}{\partial \eta_{p p}} w_{d}\left(\eta_{0}\right)
$$

Substituting (46) for $w$, we get

$$
\left.F_{s q p}=2 F\left(\delta_{s q}+\delta_{s p}+\delta_{q p}-3 \delta_{p q} \delta_{s q}-\frac{2}{3}\right) \quad \text { (no summation }\right)
$$

The finite-polynomial approximation of $w_{d}$ has a serious defect: such $w_{d}$ corresponds to the probability density that is not positively defined ([18]). But still it is a useful simplification, since the rest of the series does not make fundamental changes to lower -order correlators.

There is a simple way to estimate the validity of cutting the third term in (46)). It is known that the Lyapunov indices must be ordered ([3, 12]):

$$
\lambda_{1}<\lambda_{2}<\lambda_{3}
$$

From (45), (47) we then have

$$
|F|<\frac{2 D}{3}
$$

This is a necessary condition for cutting the series (46) $)$. In theory of turbulence, $F$ is also required to be positive to provide the right sign of energy dissipation (which is associated with the third-order correlator of $A$ ).

\section{Statistics of the T-exponential}

In the previous section we discussed different moments of the matrix $X$. Now we proceed to the averages of its exponentials (7). As we mentioned above, the matrix $R$ (which is the $T$-exponential of $\theta$ ) separates from the other variables (8) . The calculation of $z$ (which 
is, roughly speaking, the T-exponent of $\zeta$ ) is simplified by the fact that $\zeta^{N}=0$, so all the series are finite. Here we analyze more accurately the statistics of $d$. According to (7),

$$
d_{s}(t)=\exp \left(\int_{0}^{t} \rho_{s}(\tau) d \tau\right)=\exp \left(\int_{0}^{t} X_{s s}(\tau) d \tau\right)
$$

Thus, its moments are

$$
\left\langle\left(d_{s}(t)\right)^{n}\right\rangle=\left\langle\exp \left(n \int_{0}^{t} X_{s s}(\tau) d \tau\right)\right\rangle
$$

These characteristics are important in the applications; in turbulence, and in particular in the theory of passive scalar advection, they describe the separation of trajectories of liquid particles.

Consider the characteristic functional and the cumulant function of the $X(t)$ process:

$$
Z^{(X)}[\eta(t)]=\exp \left(\int_{0}^{T} w^{(X)}(\eta) d t\right)=\left\langle\exp \left(\int_{0}^{T} \operatorname{tr}(\eta(t) X(t)) d t\right)\right\rangle
$$

According to (22), (23), (25) they are related to those of the process $A(t)$ by

$$
Z^{(X)}[\eta(t)]=Z\left[\eta(t)+\eta_{0}\right] Z^{-1}\left[\eta_{0}\right], \quad w^{(X)}(\eta)=w\left(\eta+\eta_{0}\right)-w\left(\eta_{0}\right)
$$

In the previous section we saw that to calculate the moments of $X$, one can reduce $w(\eta)$ to the 'diagonal' function $w_{d}\left(\eta_{11}, \ldots, \eta_{N N}\right)$ (35). Similarly, to calculate (48), it is enough to consider

$$
\left.w_{s}^{(X)}\left(\eta_{s}\right) \equiv w^{(X)}(\eta)\right|_{\eta_{k p}=\delta_{k s} \delta_{p s} \eta_{s}, \quad \text { no summation }}
$$

Then from (32), (40), (31) it follows that $w_{s}^{(X)}$ can be expanded into the series:

$$
w_{s}^{(X)}\left(\eta_{s}\right)=\lambda_{s} \eta_{s}+\frac{D_{s s}}{2} \eta_{s}^{2}+\frac{F_{s s s}}{3 !} \eta_{s}^{3}+\ldots
$$

where the first coefficient is the Lyapunov index (34), the second is the covariation matrix, and the next coefficients correspond to higher correlators of $X$.

From (48), (49) we have

$$
\left\langle\left(d_{s}(t)\right)^{n}\right\rangle=\exp \left(w_{s}^{(X)}(n) t\right)=\exp \left(\left(\lambda_{s} n+\frac{D_{s s}}{2} n^{2}+\frac{F_{s s s}}{3 !} n^{3}+\ldots\right) t\right)
$$

We see that the moments increase exponentially with time. From (150) it also follows that in calculation of moments of the exponentials (48), one can not neglect the contributions of higher-order connected correlators even if all the moments of $X$ are dominated by nonconnected diagrams. I.e., even if the integral $\int X d t$ satisfies the condition of the central limit theorem and acts as a Gaussian, $\exp \int X d t$ is still essentially non-Gaussian. 


\section{Smoothed variables}

The cumulant function determines completely the statistical properties of a $\delta$-process. However, it cannot be measured in experiments (though the statistical averages, which are its derivatives, are measurable), nor is the probability density functional a measurable value. (Even more, the PDF is, as opposed to $w(\eta)$, badly defined in the case of non-Gaussian $\delta$-process, it requires renormalization.) In this section, we introduce smoothed random variables which can be measured in experiments. We show that they appear to have same cumulant function as the $\delta$-process, and their averages are related strongly to the correlators of $A$ and $X$.

Consider a random matrix

$$
\bar{A}=\int_{0}^{1} A(t) d t
$$

The corresponding characteristic function is

$$
z(\eta)=\langle\exp (\operatorname{tr}(\eta \bar{A}))\rangle
$$

It is related to the characteristic functional $Z[\eta(t)]$ and its cumulant function $w(\eta)$ by

$$
z(\eta)=Z[\eta \theta(1-t)]=\exp \left(\int_{0}^{T} w(\eta \theta(1-t)) d t\right)=\exp w(\eta)
$$

So, $w(\eta)$ is the cumulant function for both the stochastic process $A(t)$ and stochastic variable $\bar{A}$. The averages

$$
\left\langle\bar{A}_{i j} \ldots \bar{A}_{k p}\right\rangle=\frac{\partial}{\partial \eta_{i j}} \ldots \frac{\partial}{\partial \eta_{k p}} \exp (w(0))
$$

can be obtained from the corresponding correlators (30) by omitting the delta-functions, the terms

$$
\left\langle\bar{A}_{i j} \ldots \bar{A}_{k p}\right\rangle_{c}=\frac{\partial}{\partial \eta_{i j}} \ldots \frac{\partial}{\partial \eta_{k p}} w(0)
$$

are equal to the coefficients of the connected correlation functions.

One can express $w(\eta)$ by means of the probability density $p(\bar{A})$ : from (151), (52) one can easily derive

$$
w(\eta)=\ln \int d \bar{A} p(\bar{A}) \exp (\operatorname{tr}(\eta \bar{A}))
$$

For example, if $A(t)$ is a Gaussian process (36) then $p(\bar{A})$ is Gaussian, too:

$$
p(\bar{A})=\exp \left(-\frac{1}{4} \bar{A}_{i j} D_{i j k p}^{-1} \bar{A}_{k p}\right)
$$


and corresponds to the same cumulant function (37). In the general case, $p(\bar{A})$ can be written in the form

$$
p(\bar{A})=\exp \left(-\frac{1}{4} \bar{A}_{i j} D_{i j k p}^{-1} \bar{A}_{k p}-V(\bar{A})\right)
$$

where the function $V(\bar{A})$ corresponds to the non-Gaussian part. Then the cumulant function is

$$
w(\eta)=\ln \left(\exp \left(-V\left(\frac{\partial}{\partial \eta}\right)\right) \exp \left(\eta_{i j} D_{i j k p} \eta_{k p}\right)\right)
$$

where $\exp \left(-V\left(\frac{\partial}{\partial \eta}\right)\right)$ should be understood as a formal series. This allows, in particular, to restore the cumulant function by known coefficients in connected correlators. Now as we have the smoothed variables, the cumulant function is not necessary: $p(\bar{A})$ contains all the information about the delta-correlated random process $A(t)$, and

$$
\begin{gathered}
\left\langle X_{i j}\left(t_{1}\right) \ldots X_{k p}\left(t_{n}\right)\right\rangle_{c}=N \int d \bar{A} \bar{A}_{i j \ldots} \bar{A}_{k p} p(\bar{A}) \exp \left(\operatorname{tr}\left(\eta_{0} \bar{A}\right)\right) \delta\left(t_{1}-t_{2}\right) \ldots \delta\left(t_{1}-t_{n}\right) \\
N=\left(\int d \bar{A} p(\bar{A}) \exp \left(\operatorname{tr}\left(\eta_{0} \bar{A}\right)\right)\right)^{-1}
\end{gathered}
$$

In particular,

$$
\lambda_{s}=N \int d \bar{A} \bar{A}_{s s} p(\bar{A}) \exp \left(\operatorname{tr}\left(\eta_{0} \bar{A}\right)\right)
$$

\section{Conclusion}

In this paper we present a very simple technics to calculate the averages of time-ordered exponentials of random $N \times N$ matrices (2) and, thus, describe the evolution of linear stochastic systems.

The simplification comes from changing the variables (9), (6) that allows to separate the rotations of the eigenvectors and therefore to 'stabilize' the rest of the solution. The formal solution (2) can then be rewritten as a functional integral (11) with a simpler 'rotational' T-exponent (10).

If the random process is isotropic, the 'rotational' part can be excluded, and the averages (11) become functional integrals without time-ordered products. In the case of $\delta$ correlated in time process we calculate the Lyapunov spectrum (34) and other correlation characteristics of the T-exponent (31).

For Gaussian probability distribution of the matrices, the results coincide with those obtained earlier by [4] the non-Gaussian case is analysed in details. We also describe 
the relation of the statistics of the T-exponentials to the statistics of 'smoothed' variables. They appear to be determined by same cumulant function, so that, measuring probability density function (or correlators) of the 'smoothed' matrices, one can easily calculate, e.g. the Lyapunov spectrum.

The important feature of the non-Gaussian probability distribution is that the higherorder connected correlation functions cannot be neglected when counting the exponential averages, even if the difference from the Gaussian is small and higher-order correlators of the exponent are dominated by the Gaussian (non-connected) contribution. One more difference from Gaussian is that the Lyapunov spectrum of a non-Gaussian process is, generally, asymmetric. This is very important for applications. In particular, in [13] the observed scaling space distribution of velocity and statistical properties of a turbulent flow were derived from the statistics of velocity deformation tensor at large scales based on a stochastic analog to Euler equation.

It was shown that symmetry of the Lyapunov spectrum corresponds to time invariance of the flow, and hence must be broken in real flows. The right sign of energy flux (from larger to smaller scales in $3^{d}$ ) requires $\left\langle\operatorname{tr} A^{3}\right\rangle<0$. Thus, one cannot restrict the consideration by Gaussian approximation: non-Gaussianity is of crucial importance. The results achieved in this paper allow to calculate the Lyapunov spectrum based on experimental measurements of large-scale velocity statistics, then derive the statistic characteristics of velocity field at small scales in accordance with [13], and compare them to the observations.

This work is supported by the RAS program 'Nonlinear dynamics in mathematical and physical sciences'.

\section{References}

[1] H. Furstenberg, 1963, Trans. Amer. Math. Soc, 108, 377

[2] V. N. Tutubalin, 1978, Theory Probab. Appl. 22, 203

[3] A.V. Letchikov 1996, Russian Math. Surveys, 51, 49

[4] A. Gamba, I. Kolokolov, 1996, J. Stat. Phys. 85, 489

[5] A. Gamba 2003 J. Stat. Phys., 112, Nos. 1/2, 193 
[6] Zeldovich, Ya. B., A. Ruzmaikin, S. Molchanov, and V. Sokolov, 1984, J. Fluid Mech. 144, 1

[7] A.N. Kolmogorov 1941, Dokl.Akad.Nauk SSSR 32, 19 (reprinted in 1991, Proc. R. Soc. Lond. A 434 15)

[8] U. Frisch, "Turbulence: The legacy of A.N. Kolmogorov", Cambridge Univ. Press, Cambridge, 1995.

[9] K.P. Zybin, V.A. Sirota 2013 Phys.Rev. E 88, 043017

[10] Il'yn A.S. , Zybin K.P. 2015, Phys. Lett. A, 379650

[11] Balkovsky, E., and A. Fouxon, 1999, Phys. Rev. E 60, 4164

[12] G. Falkovich, K. Gawedzki, M. Vergassola, 2001, Rev. Mod. Phys. 73, 913

[13] K.P. Zybin, V.A. Sirota 2015, Sov.Phys.Uspekhi in press

[14] Andrew OOI, Jesus Martin, Julio Soria, M.S. Chong 1999, J. Fluid Mech, 381141

[15] N.N. Bogoliubov, D.V. Shirkov 1959 Introduction To the Theory of Quantized Fields

[16] Gantmacher P., Matrix Theory, Nauka, 1967

[17] V. I. Klyatskin 2005 Dynamics of Stochastic Systems, Elsevier

[18] Marcinkevich J., Math. Zeitschrift, 1938 44, H.4, 612 